\title{
La médecine d'urgence pédiatrique, un monde de possibilités
}

\author{
Niranjan Kissoon, MD*; Ran D. Goldman, $\mathrm{MD}^{\dagger}$
}

ENGLISH VERSION ON PAGE 453

$\mathrm{E}^{n}$ n Amérique du Nord, la médecine d'urgence pédiatrique (MUP) a atteint sa pleine maturité, alors que dans de nombreuses régions du monde, elle n'en est qu'à ses balbutiements ou est inexistante. La maturité comporte toutefois ses attentes, qui, si elles ne sont pas satisfaites, peuvent entraver la réalisation de son plein potentiel et entraîner la sénilité précoce. Les praticiens de la MUP doivent réfléchir à ce qu'on attend de cette discipline pour qu'elle atteigne son plein potentiel.

Une vue étroite de la MUP laisse croire qu'elle a pour fonction de prendre soin des enfants dans le service d'urgence pédiatrique. L'amputation des ressources et la hausse de la demande posent des défis dans les services d'urgence pédiatrique. La pénurie de lits, la réduction des services de laboratoire, le manque de personnel et le nombre croissant de patients engendrent un stress considérable dans les services d'urgence pédiatrique en ce qui a trait au triage, au traitement et à la prestation de soins appropriés à chaque enfant.

Le service d'urgence pédiatrique fait pourtant partie intégrante du système hospitalier et œuvre en étroite collaboration avec l'unité de soins intensifs pédiatriques, le service de pédiatrie et le bloc opératoire afin d'assurer une interface transparente avec les autres surspécialités pédiatriques. Bon nombre des actes médicaux qui jadis étaient l'apanage des services spécialisés sont désormais réalisés en salle d'urgence. L'intubation endotrachéale et la sédation procédurale sont deux exemples d'actes qui étaient pratiqués par des anesthésiologistes, mais qui font dès lors partie intégrante des fonctions des services d'urgence pédiatrique. La stabilisation et la réanimation pratiquée par des professionnels compétents relevaient autrefois du domaine des intensivistes spécialisés, alors que maintenant, elles sont pratiquées tout aussi efficacement en salle d'urgence par des médecins ayant reçu une formation en MUP. La pose de points de suture en cas de lacérations et la réduction de fractures fermées sont désormais des actes routiniers pour les pédiatres-urgentologues.

L'importance d'une collaboration active entre le service d'urgence pédiatrique, l'unité de soins intensifs pédiatriques et les autres services des hôpitaux a déjà été mise en lumière'. La relation de travail étroite entre l'unité de soins intensifs et le service d'urgence n'est pas une notion purement symbolique ou romantique. En effet, selon une récente étude réalisée auprès d'adultes, un patient en salle d'urgence gravement malade qui a dû attendre 6 heures ou plus avant d'être admis à l'unité de soins intensifs a séjourné plus longtemps aux soins intensifs et a été hospitalisé pendant une plus longue période, entraînant une morbidité et une mortalité plus élevées ${ }^{2}$. En tout temps, jour et nuit, le service d'urgence pédiatrique est un pivot central du continuum de soins, du foyer à l'hôpital, pour les fournisseurs de soins et les praticiens dans des bureaux et des services d'aiguillage.

Toutefois, même cette perspective du rôle de la MUP limite l'atteinte de son plein potentiel. Sur une grande échelle, de nombreux facteurs influent sur la qualité des soins que reçoit un patient (fig.1). Bien que certains de ces facteurs semblent déborder du cadre de la MUP, nous sommes d'avis que ce n'est pas le cas. En tant que praticiens de médecine d'urgence pédiatrique, nous devrions nous investir dans tous les aspects des soins de santé, y compris la détermination des installations physiques appropriées, la réponse aux demandes des collectivités, l'examen des ressources en santé, des finances, de la technologie, de l'approvisionnement en médicaments, des directives cliniques ainsi que des aspects plus « souples »,

* Programmes de soins actifs et intensifs, département de pédiatrie, Université de Colombie-Britannique, Vancouver, BC; † Département de pédiatrie d'urgence, Hôpital pour enfants de la Colombie-Britannique, Vancouver, BC

Can J Emerg Med 2007;9(6):456-8 
mais déterminants, des soins prodigués aux enfants. Bon nombre d'entre nous sont en effet déjà actifs, à l'échelle provinciale, nationale ou internationale, dans le but d'influer sur plusieurs de ces facteurs.

Les médecins d'urgence pédiatrique devraient jouer un rôle dans le continuum des soins des patients pédiatriques gravement malades et des blessés, de la médecine préventive à la réadaptation (fig. 2). Nos patients pédiatriques ont recueilli le fruit de la défense des intérêts relatifs à la prévention des accidents et la promotion de la santé (campagnes de vaccination, prévention des noyades, réglementation sur le port obligatoire de la ceinture de sécurité et réduction de blessures sportives), défense entreprise par des pédiatres-urgentologues ayant fait preuve de leadership.

Il incombe en outre aux médecins d'urgence pédiatrique de travailler de près avec les équipes paramédicales pour assurer des soins et des pratiques de réanimation adéquats sur le terrain. L'accompagnement sur place en matière de stabilisation des blessés et l'organisation de transport sécuritaire peuvent sauver la vie des enfants les plus gravement atteints ou blessés. D'ailleurs, le service d'urgence pédiatrique gère le système de transport dans bon nombre d'hôpitaux pour enfants.

Le dénouement des événements dans les régions où le système de transport fonctionnel et son rapport avec le service d'urgence pédiatrique ne sont pas bien développés met clairement en évidence leur importance. Citons comme exemple l'état d'Andhra Pradesh, en Inde, où les services de MUP et les services de soins intensifs pédiatriques sont excellents, mais où le transport d'urgence est inadéquat, donnant des résultats pour le moins désolants : le taux de mortalité des nourrissons de moins d'un an est de $52 \%$ et celui des enfants de 1 à 5 ans est de $33 \%$. Qui plus est, près de la moitié des enfants hospitalisés meurent dans les 12 heures suivant leur admission, exposant une présentation tardive causée par des services préhospitaliers inappropriés ${ }^{3}$. L'accès à un système de transport convenable pour les enfants est reconnu comme un enjeu planétaire négligét. Dans certains cas, on a recours à des bicyclettes avec une « remorque », des tricycles avec une plate-forme, des bateaux à moteur, des charrues à bœufs, ${ }^{5}$ des taxis et des autobus ${ }^{6}$, et, dans bien des régions, le transport est simplement inexistant ${ }^{7}$. Nous devons plaider la cause pour la mise en place de meilleurs systèmes de transport qui assureront une arrivée stable et en sécurité des enfants à l'hôpital.

Il faut aussi donner plus de force à la défense des droits en matière de ressources (p. ex., personnel, immeubles, équipement, etc.) pour s'assurer que le triage et les traitements en service d'urgence pédiatrique sont adéquats, non seulement dans les centres de soins pédiatriques de troisième ligne, mais aussi dans les établissements en régions éloignées afin que tous les enfants puissent recevoir

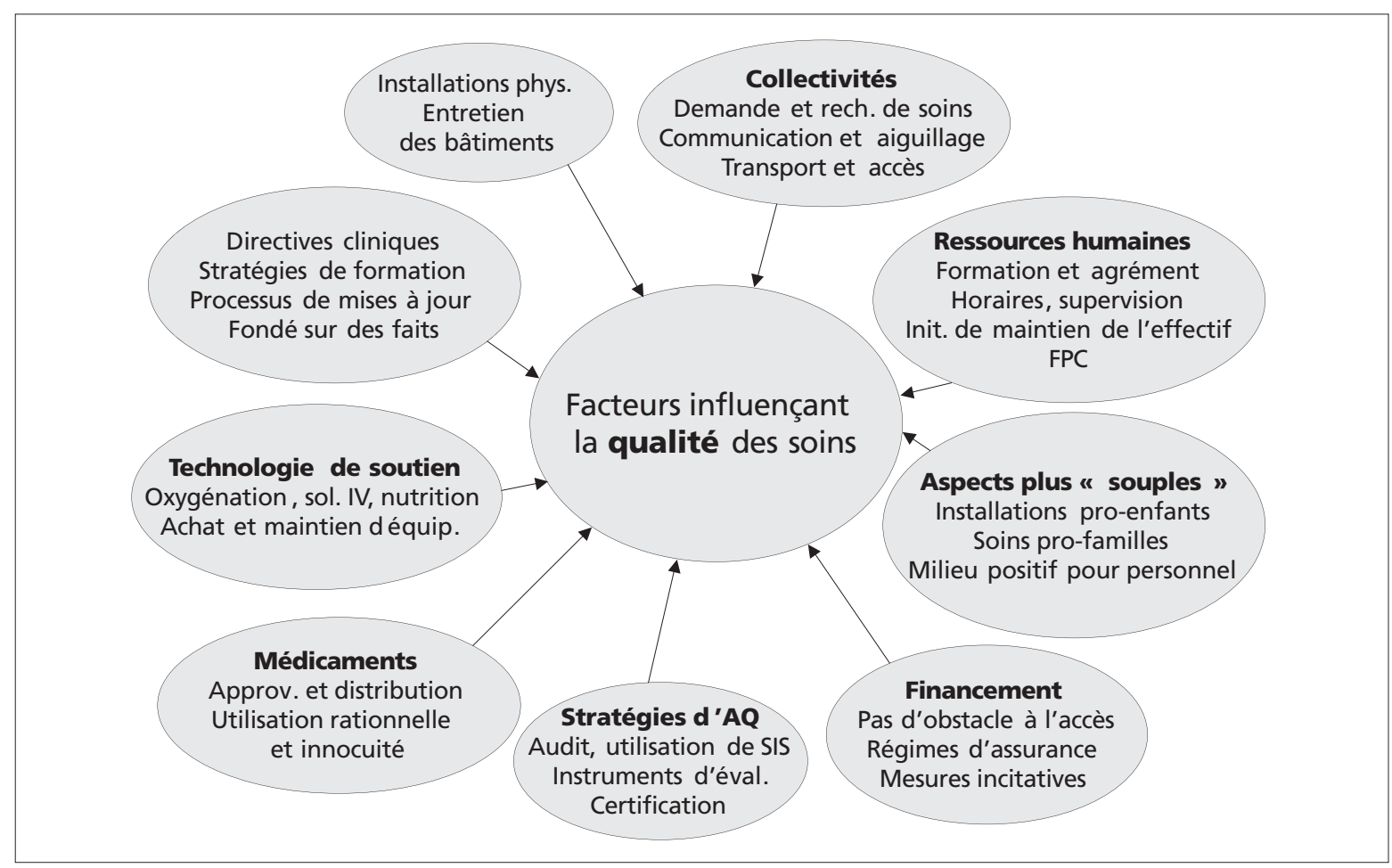

Fig. 1 : Facteurs influençant la qualité des soins. FPC = formation professionnelle continue; sol. IV = solutions intraveineuse; SIS = système d'information sur la santé; $\mathrm{AQ}$ = amélioration de la qualité 
la même qualité de soins. On ne peut insister suffisamment sur l'importance d'établir un réseau dynamique comprenant la télémédecine et la participation aux programmes d'enseignement dans notre sphère d'influence, tant à l'échelle provinciale que nationale.

Les médecins d'urgence pédiatrique devraient également jouer un rôle pivot dans les programmes de formation, et ce, avec toutes les parties, à savoir les étudiants, les résidents, et les médecins en pratique. De plus, il est crucial de voir à la formation des fournisseurs de soins paramédicaux tels que le personnel infirmier, les inhalothérapeutes et le personnel paramédical, pour la prestation de soins aux enfants. Les médecins d'urgence pédiatrique ont fait preuve de leadership à l'échelle nationale dans des secteurs de l'enseignement tels que la tenue de cours en soins avancés de réanimation des polytraumatisés (ATLS), en soins avancés de réanimation pédiatrique (APLS) et en réanimation pédiatrique avancée (PALS) ainsi que des cours de gestion des voies aériennes en réanimation et des programmes de simulation. Ils doivent aussi assumer le rôle de formateur des équipes de gestion des ressources dans les centres éloignés pour qu'elles se préparent au traitement des enfants gravement malades dans les centres de soins de troisième ligne. Les connaissances de ces médecins doivent aussi être mises à contribution dans la planification locale, régionale et nationale, de mesures d'urgence en cas de catastrophe, la préparation aux pandémies et les questions d'ordre toxicologique particulières aux enfants. Le service d'urgence pédiatrique revêt une importance encore plus cruciale dans ces situations de crises, puisqu'il constitue un point de premier contact pour la plupart des personnes qui ont besoin de soins actifs. Le service d'urgence pédiatrique devrait faire partie intégrante du processus de planification rationnelle en cas de survenue d'événements fâcheux ou de sinistres, dans toutes les collectivités. Les connaissances ainsi acquises peuvent, en temps de crise, ultérieurement s'appliquer à des situations dans le monde entier.

On peut apporter des contributions significatives au chapitre de la prévention, de l'évaluation et du traitement grâce à l'expérience acquise dans des conditions courantes. Le service d'urgence est une terre fertile pour répondre à des questions sur l'asthme, les détresses respiratoires, le croup, l'épiglottite, les traumatismes, les empoisonnements, la réanimation cardiopulmonaire et les études épidémiologiques portant sur la prévention des accidents. Le PERC (Pediatric Emergency Research Canada) au pays $^{8}$ et le PECARN (Pediatric Emergency Care Applied Research Network) ${ }^{9}$ aux États-Unis sont des exemples de participation à des projets de recherche.

Demander aux médecins d'urgence pédiatrique de

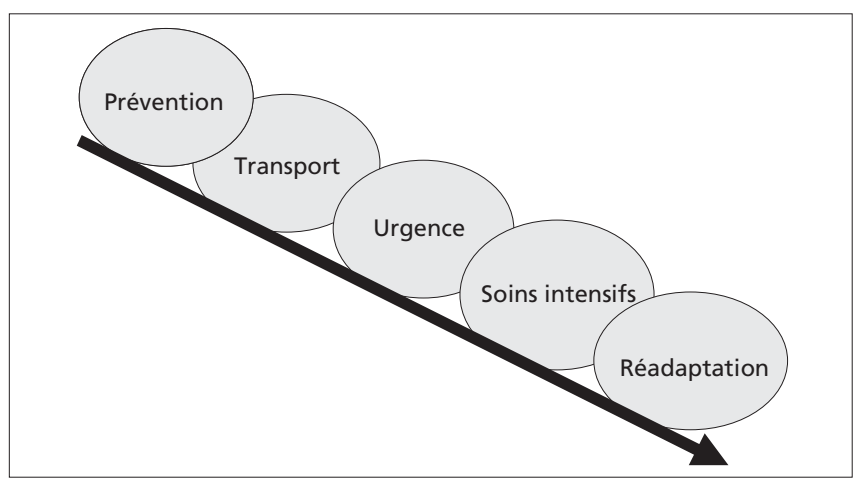

Fig. 2 : Le continuum des soins de l'enfant gravement malade.

contribuer à tant de secteurs divers peut sembler un défi de taille. Cette situation reflète cependant l'avancement de la surspécialité et l'importance capitale qu'elle joue dans la communauté médicale ainsi que dans la société dans son ensemble. Au final, le rôle du service d'urgence pédiatrique ne devrait pas être confiné dans les murs des établissements ou se limiter par notre imagination. Le service d'urgence pédiatrique devrait accueillir à bras ouverts la vaste gamme de possibilités qui s'offrent pour améliorer la vie des malades et des blessés, peu importe où ils se trouvent.

\section{Références bibliographiques}

1. Kissoon N. The pediatric emergency department/pediatric intensive care unit interface: "the double door mentality. Pediatr Emerg Care 2006;22:613-5.

2. Chalfin DB, Trzeciak S, Likourazos A, et al. Impact of delayed transfer of critically ill patients from the emergency department to the intensive care unit. Crit Care Med 2007;35:1477-83.

3. Ranganath D, Rao PS, Beligere N, et al. Under five mortality (UFM) in a pediatric intensive care unit (PICU) serving urban and rural population in India. E-PAS 2007:618418.8.

4. Duke T. Transport of seriously ill children: a neglected global issue. Intensive Care Med 2003;29:1414-6.

5. Razzak JA, Kellermann AL. Emergency medical care in developing countries: is it worthwhile? Bull World Health Organ 2002;80:900-5.

6. Mock CN, Tiska M, Adu-Ampofo M, et al. Improvements in prehospital trauma care in an African country with no formal emergency medical services. J Trauma 2002;53:90-7.

7. Nolan T, Angos P, Cunha AJ, et al. Quality of hospital care for seriously ill children in less-developed countries. Lancet 2001;357:106-10.

8. Pediatric Emergency Research Consortium Canada (PERC). Disponible sur : www.perc.med.ualberta.ca (Consulté le 17 octobre 2007).

9. Pediatric Emergency Care Applied Research Network (PECARN). Disponible sur : www.pecarn.org/ (Consulté le 17 octobre 2007).

Correspondance à : $\mathrm{D}^{\mathrm{r}}$ Niranjan Kissoon, Université de la ColombieBritannique, hôpital pour enfants, pièce K4-105, 4480, rue Oak, Vancouver BC V6H 3V4; nkissoon@cw.bc.ca 